the genome are DNA methylation and histone proteins. Unlike animals, in which the epigenetic status is updated in each generation, in plants, epimutations can be steadily inherited, and therefore are important for evolution. Salinity is the most important abiotic stress for plants. Along with morphological changes as a result of the action of a high concentration $(150 \mathrm{mM})$ of $\mathrm{NaCl}$ on tobacco regenerants (Nicotiana tabacum L.), changes in the ultrastructure of cell compartments in plant tissues, in particular nuclei and mitochondria, were observed. The mutual transformation of heterochromatin and euchromatin is primarily due to modifications of the histone proteins and the main modifications are the methylation of lysine residues. The presence of $\mathrm{NaCl}$ leads to an increase in the expression of the lysine methyltransferase gene - SUVR3, methylated $\mathrm{H} 3 \mathrm{~K} 9$, and decreased in the expression of the ASHR3 gene encoding the lysine methyltransferase, methylated H3K4. Sodium chloride causes an increase in the expression of the gene SNF2, an actin-dependent chromatin remodulator, 2 times. Proteins of the SNF2 complex untie the bonds between DNA and histones, increasing access to DNA. De novo methylation in plants is carried out by the unique enzyme DRM2, which is absent in animals. A high salt concentration causes an increase in the expression gene of DNA methyltransferase - DRM2 by $30 \%$. Moreover, the expressions of DNA-supporting methyltransferases genes remains almost unchanged. The short tetrapeptide AlaGluAspLeu (AEDL) at a concentration of 10-8M significantly stimulates the growth and development of tobacco calluses Nicotiana tabacum. Significant differences in the packing of chromatin in the presence of AEDL were detected both in normal conditions and under the action of $\mathrm{NaCl}$. Significant differences in the packing of chromatin in the presence of AEDL were detected both in normal conditions and under the action of $\mathrm{NaCl}$. In the presence of tetrapeptide, a significant decrease in the expression of genes of the SNF2 family is observed, the level of gene expression genes of methyltransferase DNA remains practically unchanged, the expression of histone methyltransferase genes also remains unchanged or decreases even. In the presence of the tetrapeptide AlaGluAspLeu and sodium chloride together, the expression of the lysine methyltransferase gene, SUVR3, increases, as in the case of the presence of sodium chloride only. An almost 3-fold increase in expression of the SNF2 chromatin modulator, which encodes an ATP-dependent helicase, which is necessary for DNA methylation, was also noted. Such an increase in the expression of chromatin remodulator is accompanied by an increase in the expression of DRM2 almost 3 times.

The study was performed in the framework of the state assignment AAAA-A17-117091460012-8.

doi: http://dx.doi.org/10.7124/bc.0009DE

\section{F-2. Probing the chromatin structure of ribosomal DNA using extended chromatin fibers}

\author{
M. Franek ${ }^{1}$, K. Kutashev ${ }^{1}$, J. Fajkus ${ }^{1,2}$, \\ M Dvořáčková 1,2 \\ ${ }^{1}$ Central European Institute of Technology (CEIT- \\ EC) and Faculty of Science of Masaryk University, \\ Kamenice 5, 62500 Brno, CZ; ${ }^{2}$ Institute of Bio-
}


physics, The Czech Academy of Sciences, Královopolská 135, 61265 Brno, CZ

357550@mail.muni.cz

The chromatin organization of ribosomal genes, which encode essential 25S, $18 \mathrm{~S}$ and 5.8S ribosomal RNA, remains unresolved to this day in many biological models. Hundreds of rDNA copies exist in the genome in a mostly tandem, head-to-tail arrangement. Nucleoli, the main transcription factories of the cell, are known to assemble around actively transcribed rDNA copies. Evidence from electron microscopy studies shows a subset of actively transcribed genes which appear mostly nucleosome-free [1], but this represents only a small fraction of rDNA units. Our understanding of the epigenetic state of the remaining copies is still incomplete [2]. Our main goal was to identify individual rDNA chromatin fibers and probe their chromatin structure. Methods We prepared chromatin fibers from the plant model Arabidopsis thaliana in combination with immunofluorescence and FISH detection of histone marks in rDNA loci. Results We show the presence of histones and histone modifications in rDNA clusters, even in plants with a reduced number of rDNA copies [3]. This indicates that a subset of rDNA copies exists in a poised state for transcription, marked by the presence of $\mathrm{H} 3 \mathrm{~K} 4 \mathrm{me} 3$ and $\mathrm{H} 3 \mathrm{~K} 27 \mathrm{me} 3$ modifications. Furthermore, these histone modifications are found intermingling or colocalizing on individual chromatin fibers in plants, reinforcing the notion that as opposed to mammalian models, H3K27me3 is not a marker of compact heterochromatin in plants. Conclusions: Microscopy of chromatin fibers has been used previously to study the structure of centromeres. We show that it is possible to adapt this strategy to study the epigenetic structure of rDNA. Once optimized, this method can be used to study repetitive genomic regions, telomeres and satellites included, and fill the gaps in knowledge in genomic regions hard to analyze by ChIP-seq.

Fundings: This project was supported by The Czech Science Foundation, grant number 19-11880Y and Ministry of education, youth and sports INTER-COST LTC18048.

References: 1. Miller OL, Beatty BR. 1969. Visualization of Nucleolar Genes. Science, 164: 955-957. 2. Dvořáčková M, Fojtová M, Fajkus J. 2015. Chromatin dynamics of plant telomeres and ribosomal genes. Plant J. 83:1837. 3)Pavlištová V, Dvořáčková M, Jež M, Mozgová I, Mokroš P, Fajkus J. 2016. Phenotypic reversion in fas mutants of Arabidopsis thaliana by reintroduction of FAS genes: variable recovery of telomeres with major spatial rearrangements and transcriptional reprogramming of $45 \mathrm{~S}$ rDNA genes. Plant J. 88: 411-424.

doi: http://dx.doi.org/10.7124/bc.0009DF

\section{G-1. Large-scale chromatin remodelling and transcriptional deregulation on der11 following translocation in Mantle Cell Lymphoma}

Diego Germini $^{1,2}$, Fatimata Bintou Sall ${ }^{1,2}$, Diana Markozashvili1 ${ }^{1,2}$, Andrei Pichugin ${ }^{1,2}$, Valérie Camara-Clayette ${ }^{3}$, Vincent Ribrag ${ }^{3}$, Marc Lipinski ${ }^{1,2}$, Yegor Vassetzky ${ }^{1,2}$

${ }^{1}$ UMR 8126, Paris Saclay University, Paris-Sud University, Institut Gustave Roussy, CNRS, Villejuif, 94805, France; ${ }^{2}$ LIA 1066 French-Russian Joint Cancer Research Laboratory, 94805 Villejuif, France - 119334 Moscow, Russia; ${ }^{3}$ Institut de Cancérologie Gustave-Roussy, Villejuif, France germinidiego@gmail.com

Mantle cell lymphoma (MCL) is an aggressive B-cell non-Hodgkin lymphoma characterized 